\title{
She stands
}

\section{Megan Green}

She stands, like Garbo.

Silky clingy clothes.

Bandanna tied

about her hair. Cooking

eggs in the apartment.

Newspapers on a small

Formica table. 